\title{
Before L1
}

\section{A differentiated perspective on infant gestures}

\section{Ulf Liszkowski}

Communication Before Language Group, Max Planck Institute for Psycholinguistics, Nijmegen, The Netherlands

This paper investigates the social-cognitive and motivational complexities underlying prelinguistic infants' gestural communication. With regard to deictic referential gestures, new and recent experimental evidence shows that infant pointing is a complex communicative act based on social-cognitive skills and cooperative motives. With regard to infant representational gestures, findings suggest the need to re-interpret these gestures as initially non-symbolic gestural social acts. Based on the available empirical evidence, the paper argues that deictic referential communication emerges as a foundation of human communication first in gestures, already before language. Representational symbolic communication, instead, emerges as a transformation of deictic communication first in the vocal modality and, perhaps, in gestures through non-symbolic, socially situated routines.

Keywords: prelinguistic communication, pointing, representational gestures, infant

\section{Before L1}

Language is a hallmark of modern human communication, but language is maybe best understood as an emergent property over historical time of more basic and non-linguistic yet unique forms of human communication. Language, whether spoken or signed, is a conventional code, but the code alone is not a sufficient basis for communication. For example, the statement "This is a concert" to a person whose mobile phone goes off in the middle of a symphony is meant to communicate much more than what is actually coded linguistically. Further, in the absence of a shared linguistic code speakers of different languages still communicate successfully with gestures. Even in the absence of conventionalized gestures (i.e., sign 
language) deaf-born humans who are deprived of spoken and signed linguistic codes can still communicate successfully with creatively invented 'home-sign' gestures (Goldin-Meadow, 2003; Senghas, Kita, \& Özyürek, 2004). The main point is that language, albeit special in itself, is only the tip of the iceberg of human communication, with all its complexities below the surface still waiting to be discovered.

Human communication is an inferential process. A recipient tries to understand a sender's intention, and the sender, in turn, knows this and intends the recipient to understand his intention (Grice, 1957; Sperber \& Wilson, 1986). This recursive inferential model of human communication involves two main psychological components. First, social-cognitively, interlocutors need to form and understand intentions toward others' intentions and understand epistemic states to transmit and infer referential content. Second, motivationally, human communication at its base is cooperative. The sender marks and formulates his utterances so that the receiver understands them, and the receiver tries to understand them as the sender intended her to. Communication takes place in a joint zone ('common ground'; Clark, 1996). Communicative attempts outside the zone fail to communicate successfully, whether they are coded linguistically or not. Further, humans communicate with cooperative motives, for example to freely provide others with relevant information, not only to gain immediate direct benefit. Human communication indeed involves complex social-cognitive and cooperative abilities, abilities which must develop somehow.

Animal communication provides an informative contrast to the inferential and cooperative human communication model. Most of animals' signals are reactions to stimuli, usually with little flexibility, often lacking referential or even communicative intent (e.g., Call \& Tomasello, 2007). Dawkins and Krebs (1978) emphasized that ritualized combats, mating, courtship and so forth really are individualistic behaviors to maximize the benefit of the individual who emits the signal by literally using another individual's muscles from afar. Animal communication thus seems to involve quite different processes compared to human communication. It mostly lacks a cooperative structure and is rather based on individualistic attempts of manipulating the environment to one's own benefit, whether the environment is animate or inanimate. How and when in phylogeny cooperative behaviors evolved is currently a topic of hot debate (e.g., Boyd, 2006). It would seem that it is only with the advent of cooperative motives that we would first see rudimentary forms of communication proper that go beyond the manipulation of others' muscles for one's own direct benefit.

Infant gestural communication is a test case for models of communication. If language rests on some more fundamental yet already unique forms of human communication, the core infrastructure of human communication should already 
be present before language, and already be different from other species. This paper takes an ontogenetic perspective on human communication that is largely independent of language and linguistic code models by investigating infant gestures before acquisition of a first language (L1). In contrast to adult gesture research, it does not address speech-accompanying gestures as part of or a complement to an existing linguistic system, but instead focuses on the emergence and use of core gestures before any speech or linguistic system has yet developed. The main question is: To which extent do infant gestures already share a common cognitive and motivational infrastructure with fully-fledged adult communication before language has emerged? From a developmental perspective, a related question is: Where do infant gestures originate from ontogenetically?

With regard to the gestural origins, two perspectives may broadly be distinguished. Classically, from a language acquisition perspective, infants' gesturing has been interpreted as a kind of social tool-use, building on infants' emerging intentionality (Bates, 1979). Infants' intentionality, in turn, supposedly originates from infants' individualistic sensorimotor schemes toward the physical environment. Infant gesturing, on this account, is a tool for individualistic problem-solving serving one's own benefit, which rests on the emergent intentionality from individualistic object-directed action schemes. On this account, however, it is not clear to which extent infant gestures already reflect a psychology of intentions toward others' intentional states, and cooperative motives to commune with and achieve mutual understanding involving benefits for the other. It is also not straightforward how inferential-cooperative communication would simply emerge on the heels of individualistic object-directed action schemes and egocentric motives.

Other accounts instead emphasize humans' ultra-sociality as the origins of infants' gestures. Infants are entrenched in rich interactional contexts with competent adult communicators from the beginning, which provide a strong basis for the ontogenetic origins of cooperative human communication (Bruner, 1983; Werner \& Kaplan, 1963). Infants are attuned to people from birth (e.g., their faces and voices) and an attachment system assures adults' interest in interacting with their offspring on a psychological level beyond mere nurturing (Bowlby, 1969). Very young infants are already sensitive to several interaction cues like ostension and reference (see Csibra \& Gergely, 2006), and to the contingencies of turn-taking as evidenced, for example, by their gaze aversion and reengagement behavior in the still-face paradigm (an adult interrupts an ongoing face-to-face interaction with a still face; see Adamson \& Frick, 2003). Cognitively, however, on this account it is not so clear to which extent infant gestures are already under their control and intentionally directed at people.

One perspective on the emergence of gestures thus emphasizes infants' developing intentionality from individualistic object-directed sensorimotor schemes as 
the key feature in the emergence of human communication. In that perspective, the origins are individualistic and the underlying motivation egocentric and still lacks the cooperative structure of adult communication. Another perspective instead suggests that the roots of human communication are a primary motive for social contact within an ultra-social environment. It is less clear on this account, however, whether infants' behaviors already involve communicative and referential intent, or whether adults only interpret and construct them as communicative. Few empirical studies have directly tested the underlying complexities of infants' gestures with regard to communicative intent, social cognition and cooperative motives. In what follows, I first present recent findings on infants' referential deictic gestures, in particular infant pointing. These findings constitute evidence for social-cognitively and motivationally 'rich' prelinguistic referential communication. Next, I review relevant findings on infants' representational gestures emerging after pointing. These findings yield little support for a symbolic interpretation of infant gestural communication, in particular not before language. Instead, I propose a leaner re-interpretation of these gestures as non-symbolic gestural social acts.

\section{Infant gestures}

In infancy research infants' gestures have been operationally defined as intentionally communicative based on infants' (1) looks to the adult, (2) persistence and flexibility to achieve a goal, and (3) conventionalization of behavioral forms (Bates, 1979). Intentionally communicative gestures have been classified into deictic and representational gestures (Bates, 1979). Deictic gestures show or present a referent in the environment (deixis, Greek "to show"), the most prominent gesture being pointing. Deictic gestures are thus used to communicate referentially. Representational gestures re-present a referent, either in a conventionalized arbitrary form with a gesture that is commonly associated with the idea it should trigger (e.g., thumbs up for 'good'), or in an iconic way by miming a referent or pretending to act out the content of a message (e.g., raising fist half open toward mouth for 'drinking'). Representational gestures are thus used to communicate referentially by means of a symbolic vehicle which represents the referent.

\section{Deictic gestures and infant pointing}

Classically, infant gestures such as showing, giving, reaching, and pointing have been classified as deictic gestures (Bates, 1979). In fact, infant reaching may better be described as a request rather than a showing gesture which is ritualized from abbreviated grasping attempts, maybe similar to begging gestures in apes 
(Call \& Tomasello, 2007). However, infants from around 9 months also pick up objects and hold them out with an outstretched arm, usually to the delight of their caregivers who then comment on them. Although infants are often less ready to let an adult take the object they are holding out (and so such 'showing' is not yet an 'offer'), infants will sometimes also hand over objects, often by placing them in the parents' lap or hands. Such 'placing' is deictic in the sense that the object becomes a referent by virtue of the specific place where it is put (Clark, 2003). In a sense, these gestures are thus referential because they bring specific objects to the attention of others. Further, they seem to be motivated cooperatively, to mutually engage about things. Showing and placing are thus good candidates for crediting infants with intentional deictic referential communication and may reflect foundations of uniquely human communication.

However, it is not clear precisely how these gestures work from the infants' point of view. There are no experiments to my knowledge which have directly tested referential intent underlying infants' showing or placing. Since these gestures involve objects at hand, a leaner interpretation is that they originate from individualistic object-directed actions. For example, infants may shake objects as an exploratory activity, while parents interpret this as communicative object exposure. Based on parents' reactions the activity then becomes ritualized into a social gesture. Once ritualized, social gestures may be interpreted as intentional communication. However, they need not yet be intentionally deictic and express referential intent on the infants' part. Instead, showing and placing may simply reflect a way of interacting with others, non-referentially. These gestures are motivationally interesting because they afford and establish social contact. The underlying communicative and cognitive complexities, however, are not yet clear.

Pointing emerges after showing and placing around 12 months. Pointing is interesting because it enables reference to things at a distance and does not require physical contact with objects. Its action scheme has no function outside communicative contexts, in particular not for individualistic actions on objects. Pointing is even used to refer to referents beyond the immediate perceptual 'here and now' as one can point to a chair to refer to the late grandfather who used to sit in it. Referring to entities displaced from the 'here and now' is clearly a distinguishing feature of human communication. Social-cognitively, communicating by pointing requires an understanding that people attend to things and that one can direct their attention to these. It also involves an understanding of the shared background against which the point's referent must be interpreted. Motivationally, adults point with cooperative motives, for example, to engage about things together, or to help others notice what they need to know. Two core aspects of human communication, that is, social cognition and cooperation, are thus already reflected in the 
single special act of human pointing. But developmentally, we need to know how this gesture works from the point of view of prelinguistic infants.

In a series of recent experimental studies my colleagues and I have investigated infant pointing when it has just emerged around 12 months. These studies were designed to challenge communicatively 'lean' accounts. For example, developmental psychologists have proposed that (i) infants initially point non-communicatively (Desrochers, Morissette, \& Ricard, 1995); (ii) pointing is non-referential as it does not involve a social-cognitive understanding of recipients' attention (Moore \& D'Entremont, 2001); and (iii) infants' motivation is mainly egocentric, to obtain objects or attention to the self (Bates, Camaioni, \& Volterra, 1975; Moore \& D'Entremont, 2001; Gomez, Sarria, \& Tamarit, 1993). We used different procedures to elicit infant pointing. Either interesting events happened (a light flashed; a puppet appeared from behind a curtain), or an adult searched for something she needed, or the infant desired something out of reach. What we systematically varied in all these studies was the social context of infant pointing.

First, with regard to communicative intent, findings were that already at twelve months infants use their pointing gestures to communicate. For example, when a recipient did not react to their pointing, infants persisted in their communicative goal and augmented the signal as reflected in repeated pointing and increased vocalizations compared to a situation in which the adult typically reacted by sharing attention and interest (Liszkowski et al., 2004, 2007a). Even more clearly, before infants initiated a point, they considered whether the recipient attended to them and so could see their point. When an adult turned sideways and did not look at infants (and so could not possibly see a point), infants pointed less than when the adult was turned toward them and so could see and react to their visual gesture (Liszkowski, Albrecht, Carpenter, \& Tomasello, 2008). These experimental results thus establish that 12-month-olds point with communicative intent.

Second, with regard to reference, we found that infants point fully referentially, making reference even to absent entities. In two studies a recipient misidentified infants' referents and either attended solely to the infants' face, or to an irrelevant object nearby the intended referent. In both these cases of referential misunderstandings, infants attempted to redirect the recipient's attention by repeating their pointing to their intended referent more often than when the recipient had correctly identified the referent (Liszkowski et al., 2004, 2007a). Infants thus point to refer to particular entities. In new studies, we found that infants even referred to previous events or objects which were not present at the moment of testing. For example, when infants had attended to an interesting event and it had ceased, they then pointed to its previous, now-empty location depending on how a recipient had reacted to it before (Liszkowski et al., 2007b). Further, to obtain a desirable object that was absent at the moment of request, infants but not chimpanzees who 
were tested in the same study design pointed to the object's usual but now-empty location, thus referring to the absent entity (Liszkowski, Schäfer, Carpenter, \& Tomasello, submitted). The results thus establish that infants point to refer others to specific, and sometimes even absent referents.

In further studies we tested infants' epistemic understanding underlying their referential pointing. We found that infants pointed significantly more often to an interesting event when the adult had not yet seen it than when she already had (Liszkowski et al., 2007b). Moreover, we established that infants point to inform an adult who is looking for an object (Liszkowski, Carpenter, Striano, \& Tomasello, 2006). In this new search paradigm, an adult lost track of one of two boring objects she (but not the infant) needed, and then searched around with a quizzical look. Infants readily pointed out the object the adult needed, without requestive accompaniments or personal interest in them, and more often when the adult was ignorant than knowledgable of the objects' locations (Liszkowski, Carpenter, \& Tomasello, submitted). These results thus reveal that infants point referentially with an understanding of the attentional and epistemic states of others.

Third, we found that infants point for others with cooperative and prosocial motives. The studies show that infants point at interesting events to share their interest about these with others. For example, when an adult only oriented to the infant's referent but then did not comment on it (Liszkowski et al., 2004), or when the adult's comment about a referent was unenthusiastic and therefore did not match the infant's interest (Liszkowski et al., 2007a), infants were dissatisfied, as reflected in their differential pattern of pointing. Crucially, when infants already shared an attentional focus with the adult, they then still pointed if the adult expressed interest in the referent, in order to share and express their alignment with the adult's expression of attitude (Liszkowski et al., 2007b). These findings show that infants do not only want to share the visual focus on a referent, they also want to express and share their attitudes about a referent. Moreover, we demonstrated for the first time that infants also point to help others, which may be interpreted as the ontogenetically earliest evidence for altruistic helping without direct benefit for the self. In these studies, infants pointed to help an adult find things which they themselves did not request or find of particular interest (Liszkowski et al., 2006), and more so when the adult needed help to find it than when she did not (Liszkowski et al., submitted). The studies thus provide experimental evidence that infants point with cooperative and prosocial motives, i.e., to align with and to help others.

The new experimental findings provide a new look at infant pointing as a human communicative act including full fledged reference on a mental level and cooperative motives like sharing and helping, all before language has emerged (see Tomasello, Carpenter, \& Liszkowski, 2007). This interpretation is further 
supported by the fact that infants also comprehend the pointing of others in the same way that they themselves point (Camaioni, Perucchini, Bellagamba, \& Colonnesi, 2004; Behne, Liszkowski, Carpenter, \& Tomasello, in prep.). The exact process of the emergence of pointing is not well understood at the moment (see also Lock, Young, Service, \& Chandler, 1990). Since pointing is not functional as an object-directed action (unlike, e.g., reaching), and because it is used communicatively with cooperative motives from the beginning, pointing does not seem to simply originate from individualistic object-directed actions, in particular not from reaching (see also Franco \& Butterworth, 1996). Presumably, as already suggested by Werner \& Kaplan (1963), the ability to refer originates in interpersonal contexts from an emerging motive to share objects together as 'objects-of-regard'. On that account, infants' showing and placing may enhance object-involved interpersonal contexts and, through social scaffolding, lead to infants' comprehension and production of referential communication. It is not clear whether imitation or instead a more biological basis leads to the particular form of index-finger pointing. Given the communicative complexities of pointing when it has just emerged, however, it should be conceptualized as developmental accomplishment of - and not a precursor to - referential communication.

\section{Representational gestures and infant gestural social acts}

Like words, representational gestures have semantic content and are used for symbolic reference. They are thus different from pointing which in itself does not represent in a symbolic way or carry meaning independent of its context. Infant representational gestures emerge after pointing, in the case of arbitrary gestures through imitation and, in the case of iconic gestures, also creatively from one's own action experiences (Bates, 1979; cf. also Capirci et al., 2005). Symbolic communication is a transformation of earlier forms of deictic communication which presupposes skills of reference and, in addition, cognitive skills for symbolizing. Representational gestures, especially iconic gestures, require the cognitive ability to decouple an action directed at an object from the communicative act of representing a referent with that action. Iconic gestures thus involve some kind of pretending or miming of an object-related action in order to represent a referent.

Developmentally, however, it is possible that both arbitrary conventional and iconic gestures are initially simply reproduced via imitation. To merely reproduce an iconic gesture one need not understand the 'etymological' relation to its action scheme derivate and decouple action and representation. Indeed, studies show that there is no advantage for infants' comprehension of iconic over arbitrary gestures (Namy, Campbell, \& Tomasello, 2004). It is thus possible that representational gestures initially rather reflect non-symbolic forms of participating in social 
situations, routines and game formats. On such an account, infant representational gestures may be re-interpreted as what I term non-symbolic gestural social acts.

Gestural social acts are conceptually and developmentally different from fully symbolic representational gestures and best understood in terms of their origins. They originate in social routines, games, and contingencies through interactional processes and are mainly used to do what one does with others socially. Just like objects afford certain object-directed actions, for infants social situations and persons too may afford certain social gestures. For example, a routine in which a mother sings 'we are birds' while flapping with her arms, may lead the infant to eventually flap arms too, initially just in the game context just with the mother, and then maybe as a way of initiating or maintaining contact with other social partners, out of an interest in the social world and a proclivity to interact. The point is that initially the infant need not know that the gesture is used to symbolically represent the referent 'bird' or 'bird game'. Instead of representing anything symbolically, gestural social acts are about the direct social activity and interaction itself.

It is not entirely clear what would be convincing evidence for a symbolic understanding of representational gestures, but there are several co-occurring behaviors which could support a symbolic interpretation. Clearly, one would expect generalization of usage and decontextualization (Werner \& Kaplan, 1963), since symbols are rather abstract and not bound to specific situations or recipients. Further, good evidence would be skills for creatively producing iconic gestures, which requires the cognitive ability to decouple actions from objects and, instead, use these actions to represent referents. Other supportive evidence for a symbolic interpretation of representational gestures is their combination with other deictic and representational gestures or words into symbolically communicated messages (for example, gesture for sleep + word 'bed', or gesture for sleep + point to bed). More independent support would be the cognitive ability to creatively extend pretense acts and understand symbols more generally, for example maps or scale models. In what follows I review and discuss findings on infant representational gestures which have been taken to support a symbolic interpretation. In light of the findings, I propose a leaner re-interpretation of these gestures as initially nonsymbolic gestural social acts.

Classically, Bates (1979) proposed a transition from deictic to representational communication around 13 months when infants start to use their first words and representational gestures to name things and engage in symbolic play such as putting doll shoes on a doll's feet, or stirring with a spoon to express something about 'spoonness'. Such pretense or symbolic play has been interpreted as representational gesturing ('gestural naming'). For example, Caselli (1990), based on a diary study, reported episodes of symbolic play at 9-12 months which she interpreted as communicative semantic acts similar to first words (e.g., holding empty fist to ear 
for 'telephone'). However, most of these observations were about narrowly defined, context-bound social acts learned and reproduced in specific social interactions. Further, these 'naming' gestures could be a form of individualistic pretense play instead of being communicative. Moreover, they may not even involve pretense, but instead only reflect individualistic trying and practicing of object-directed action schemes. In fact, more recent studies suggest that it is around 2 years of age that children differentiate pretense from serious trying (Rakoczy, Tomasello, \& Striano, 2004) and creatively extend others' pretense acts (for example, when an adult pretends to spill some coffee on a table, infants then pretend to clean the table; Harris \& Kavanaugh, 1993).

Acredolo and Goodwyn (1988, Study 2) conducted longitudinal interviews with parents of 16 infants beginning at 11 months over a weekly assessment period of 9 months. They concluded that infants gesture representationally from around 14 to 15 months onwards, with a possible advantage in onset of representational gestures over words of about 3 weeks (only with gesture training; Goodwyn \& Acredolo, 1993). They coded gestures if they occurred more than once (to exclude one-time off mimicking) and if they were clearly discernable from other (e.g., vocal) behaviors. They distinguished 'object gestures' denoting the presence of specific objects or events (e.g. sniffing for 'flower') if they were generalized from a real object to a picture of it or vice versa; 'request gestures' (like knob-turning for 'open door'; arms-up for 'pick me up') which were specific to situations and contexts and, as the authors noted, not generalizable; and 'attributes' which were object descriptions, like blow for 'hot' or palms up for 'all gone', if they were not instrumental.

The main findings were that request, attribute, and object gestures emerged between 14 and 15 months (in that order), and that object gestures were most frequent (38 types in $75 \%$ of the sample during 9 months of weekly observations, thus on average 2 gesture types per infant). Of these object gestures $32 \%$ originated within interactive routines, either through repeated exposure or explicit teaching. Instead, $58 \%$ of the object gestures were mimed actions without objects in hands, for example, rubbing the tummy for 'soap', panting for 'dog', or flapping arms for 'bird', which the authors argued had emerged outside interactive routines. Ten percent of the gestures depicted perceptual qualities of the referent (e.g., a cupped hand for 'moon').

However, a number of issues challenge the authors' interpretation of symbolic gesturing before language. Methodologically, parental weekly interviews may be limited with regard to issues of modes of acquisition (e.g., inside vs. outside interactive routines) and the extent of generalization. Operationally, request gestures are context-specific and ritualized abbreviated action attempts, observable also in non-linguistic apes, rather than symbolic (e.g., instead of climbing up mother's leg 
it becomes sufficient after a while to simply 'raise arms'; see also Call \& Tomasello, 2007). Further, attribute gestures also do not involve great generalization, because their occurrence is constrained to specific situations like feeding or clean-up/hiding games (e.g., when eating, mum always blows, or when things are gone one always raises palms). They are thus rather prototypical gestural social acts, used to do what others' do in specific social situations.

With regard to object gestures, infants had a very small repertoire of on average 2 object gestures. This is in stark contrast to the rapid word growth at that age. Further, object gestures were generalized only to similar referents in similar contexts rather than being flexibly used. The degree of generalization was thus fairly small. A third of these gestures emerged inside interactive routines, consistent with the re-interpretation of these gestures as being gestural social acts. With regard to the remaining gestures, it is not clear that they really emerged outside a social context, especially not as individually created iconic symbols. To be parsimonious, it seems unnecessary to assume that young infants draw an analogy between birds' wings and their own arms, then on this basis creatively mime birds by 'flapping arms', and finally use this pantomime with the intent to communicate something about a bird. Similarly, it is not clear that infants creatively produce gestures to depict perceptual qualities of objects. First, the very low frequency alone does not seem to suggest a general ability to create and flexibly communicate with iconic gestures. Second, if we imagine Acredolo and Goodwyn's infant with cupped hands, it seems unlikely that the infant would invent this sign outside a social context by looking at the moon (in a sleepless night) and then for the first time communicate about the moon by creatively inventing the sign 'cupped hands'.

In another study, Iverson, Capirci, and Caselli (1994) collected observational data from 12 infants at 16 and 20 months in 45 minute play sessions to compare the development of gestural and vocal communication with regard to deictic and representational gestures. They measured 'showing', 'pointing', and 'ritualized requesting (reach)' as deictic gestures, and as representational gestures 'predicates' (e.g., hot; tall), 'conventional gestures' (e.g., no; bye-bye; all gone), and 'nominal gestures' (cf. Acredolo and Goodwyn's 'object gestures'; e.g., drinking from a cup, demonstrated with or without the object, or flapping hands for 'birdie'). They found that most 16-month-olds communicated more frequently with gestures than vocally. However, the vast majority of all gestures at both ages were actually deictic, not representational. Moreover, the deictic gestures increased with age from $68 \%$ to $80 \%$ of all gestures, while representational gestures decreased correspondingly. Interestingly, the vast majority of deictic gestures at 16 and 20 months consisted of pointing alone (rising from about $60 \%$ to $80 \%$, chance $=33 \%$ ). Further, already at 16 months the total number of representational gestures was much smaller than the number of representational words $(25 \%$ of the number of representational 
words). In addition, with regard to the types of representational gestures, of 14 nominal gestures observed at 16 months, half were done with an object in hands, thus not being clearly distinguishable from object-directed actions.

The study shows quantitatively that infants use representational gestures rather seldom compared to pointing and verbal communication. This suggests that representational gestures - in contrast to pointing - play only a small role in infants' prelinguistic communication. Also, the number of representational gestures is much smaller than that of representational words from the outset, suggesting that infant representational communication is vocal rather than gestural throughout. Further, relative to deictic gestures, infants' low-frequent representational gesturing even decreases during the transition to symbolic communication, which suggests that infants do not build their emerging symbolic verbal communication on skills of symbolic gestural communication. Instead, pointing is the most frequent which increases even until the two-word stage relative to all other gestures (see also Lock et al., 1990). This suggests that it is actually pointing which leads infant communication throughout the prelinguistic period to the two-word stage, not representational gestures.

To investigate the role of gestures in the transition from one to two-word utterances, further research has addressed gesture-word combinations in the second year (e.g., Capirci, Iverson, Pizzuto, \& Volterra, 1996; Iverson \& Goldin-Meadow, 2005). Gesture-word combinations in these studies are, for example, instances where infants point to a cup and say 'drink', or 'cup'. The main findings in these studies are that the vast majority of gestures that co-occur with words are, in fact, pointing gestures only. Further, combinations in the gestural mode, for example, two representational gestures (gesture for cup + gesture for drink) or deictic and representational gestures (point to cup + gesture for drink) are virtually absent (see Pizzuto \& Capobianco, 2005). These findings thus suggest that in the transition to symbolic (verbal) communication, infant representational gestures do not play a significant role. Instead, they are bypassed by pointing and first word utterances. The co-occurrences of point + word may in fact also be interpreted as single holistic utterances (albeit in two modalities) instead of true combinations of two different utterances, especially since a point itself does not carry semantic content independent of the communicative context. In a sense, this may suggest that also one-word utterances are a fragile form of verbal representational communication, which initially still heavily relies on pointing.

The studies show that infants in their second year of life produce forms of representational gestures. Quantitatively, these gestures are small in number, they are low-frequent, and used much less than deictic gestures (in particular pointing) or vocal communication. Infants thus rarely use representational gestures for their prelinguistic communication. Further, there is no conclusive evidence that 
infants use these gestures symbolically, in particular not before language. Infants' representational gestures may thus be reinterpreted as non-symbolic gestural social acts. Initially, infants use these gestures as a form of social activity and a way of doing things together, mainly in play formats or routines. At this stage, infant representational gestures do not re-present anything other than the gestural activity itself. Instead, they rather present infants' joint activity directly, their social acts done with gestures.

\section{A differentiated perspective on infant gestures}

Infant gestural communication provides important insights into the emergence and nature of human communication. It is a model for unique forms of human communication independent of linguistic codes. Findings show that infants communicate with gestures already before the acquisition of a first language, in ways that are already different from those of other species. If language is the tip of the iceberg of human communication, infant gestural communication is its base. Focusing on the base of infant gestures more specifically, findings suggest a differential picture. Deictic referential gestures (i.e., pointing) are foundational to human communication. Representational gestures are an emergent property of interactional processes in the transformation of gestural deictic-referential toward verbal symbolic communication.

A new look at prelinguistic infants' pointing has revealed that infants point to communicate referentially - including referring to absent entities - in various and flexible ways, with cooperative motives and a social-cognitive understanding of the epistemic state of others. Infant pointing thus bears core features of the infrastructure of human communication, already before language has emerged. In typically developing infants, pointing is foundational to language and mediates its acquisition. In children with autism, the absence of deictic pointing is source and symptom of their impaired communication (e.g., Baron-Cohen, 1989). Nonhuman primates, apes, who do not have language, also do not point for each other (Tomasello, 2006). Gestural deictic referential communication is thus primary in the emergence of human communication, predating language both ontogenetically and, perhaps, phylogenetically. It would be interesting to know from where pointing originates. Given that infants point socially from the beginning, its motivational background is presumably rooted in interpersonal contexts. It is possible that pointing is based on earlier interactive routines and play formats which involve objects and in which infants actively participate with gestures such as showing, and give-take exchanges. 
The re-interpretation of infant representational gestures questions whether infants gesture symbolically before language, and whether they produce iconic gestures creatively from individualistic object-directed actions or, instead, acquire them socially from interaction. The findings suggest that infants rarely communicate with such gestures, in particular when compared to pointing and words. Their use is also still quite context-bound and not integrated into infants' emerging combinations of communicative utterances, quite unlike infants' use of pointing. There is only little support that such gestures emerge as creatively produced pantomimes from individualistic object-directed action schemes. Cognitively, there is also little evidence that these gestures involve symbolic understanding, which emerges in related areas like pretense and scale-model games only around 2 years of age (DeLoache, 2004). Instead, these gestures may be reinterpreted as gestural social acts which emerge in interactive routines and game formats, mainly through observation and reproduction. They involve a bi-directionality in the sense that both infant and adult know how to react when addressed, but infants presumably still use these gestures non-symbolically to initiate or maintain social interaction based on interactive routines. Gestural social acts build on infants' earlier communication skills and social-cooperative motives. However, rather than being used as a symbolic vehicle to represent a referent in conventional or iconic ways, the interaction is about the gestural activity itself. Such types of non-symbolic social gesturing may lead to the accumulation and extension of common grounds necessary for symbol acquisition. The social use suggests that gestural social acts originate in infants' motivation for social participation and interaction, not their individualistic object-directed actions.

It is not entirely clear where representational gestures originate from and what role they play in the emergence of language. Phylogenetically, one possibility is that after pointing and before spoken language, there was a phase of creating iconic gestures from action schemes. Ontogenetically, however, this is not the case. Instead, infants acquire language before they creatively produce iconic gestures, and it is deictic, not representational gestures, that play a pivotal role in the acquisition of a first language. It would be interesting to know whether infants' gestural social acts, like pointing, also mediate the acquisition of language or whether they have a more general social function, for example, in the emergence of conventionality and joint activities. It is an open question whether the absence of infant gestural social acts would hamper the transition from pointing to language in any specific way.

Based on the available ontogenetic evidence, this paper proposes a differentiated perspective on infant gestures before language. Infant pointing is already a complex prelinguistic form of human cooperative referential communication. Infant representational gestures are still a form of non-symbolic gestural social 
acts which draw on earlier interaction skills and social-cooperative motives. This perspective thus also emphasizes the interactional basis of language acquisition and symbol formation. Neither pointing nor representational gestures seem to simply emerge from individualistic object-directed action schemes. Instead, their emergence is presumably mediated by a primary motive for social contact and interaction. We need to know more about the origins of deictic gestures and about the role of gestural social acts in the transition to language to better understand the nature and origins of human communication.

\section{Acknowledgements}

I thank Malinda Carpenter, Marianne Gullberg, Kees de Bot, Susan Schmidt, and two anonymous reviewers for helpful comments on an earlier draft.

\section{References}

Acredelo, Linda \& Susan Goodwyn (1988). Symbolic gesturing in normal infants. Child Development, 59, 450-466.

Adamson, Laura \& Janet Frick (2003). The still face: A history of a shared experimental paradigm. Infancy, 4 (4), 451-473.

Baron-Cohen, Simon (1989). Perceptual role taking and protodeclarative pointing in autism. British Journal of Developmental Psychology, 7 (2), 113-127.

Bates, Elizabeth (1979). The emergence of symbols: Cognition and communication in infancy. New York: Academic Press.

Bates, Elizabeth, Luigia Camaioni, \& Virginia Volterra (1975). The acquisition of performatives prior to speech. Merrill-Palmer Quarterly, 21, 205-226.

Behne, Tanya, Ulf Liszkowski, Malinda Carpenter, Michael Tomasello. Manuscript in preparation.

Bowlby, John (1969). Attachment and Loss. Vol. 1: Attachment. New York: Hogarth.

Boyd, Richard (2006). The Puzzle of Human Sociality. Science, 314, 1553.

Bruner, Jerome (1983). Child's talk. New York: Norton.

Call, Josep \& Michael Tomasello (Eds.) (2007). The gestural communication of apes and monkeys. New York: LEA.

Camaioni, Luigia, Paola Perucchini, Francesca Bellagamba, \& Cristina Colonnesi (2004). The role of declarative pointing in developing a theory of mind. Infancy, 5 (3), 291-308.

Capirci, Olga, Annarita Contaldo, Maria Cristina Caselli, \& Virginia Volterra (2005). From action to language through gesture: A longitudinal perspective. Gesture, 5 (1/2), 155-177.

Capirci, Olga, Jana Iverson, Elena Pizzuto, \& Virginia Volterra (1996). Communicative gestures during the transition to two-word speech. Journal of Child Language, 23, 645-673.

Caselli, Maria Cristina (1990). Communicative gestures and first words. In Virginia Volterra \& Carol J. Erting (Eds.), From gesture to language in hearing and deaf children (pp. 56-67). Berlin: Springer. 
Clark, Herb (1996). Uses of language. Cambridge: Cambridge University Press.

Clark, Herb (2003). Pointing and placing. In Sotaro Kita (Ed.), Pointing: Where language, culture, and cognition meet (pp. 243-268). Mahwah, NJ: Lawrence Erlbaum.

Csibra, Gergely \& Gyorgy Gergely (2006). Social learning and social cognition: The case for pedagogy. In Yuko Munakata \& Mark H. Johnson (Eds.), Processes of Change in Brain and Cognitive Development. Attention and Performance XXI (pp. 249-274). Oxford: Oxford University Press.

Dawkins, Richard \& John Krebs (1978). Animal signals: information or manipulation? In John Krebs \& Nicolas Davies (Eds.), Behavioural ecology: An evolutionary approach (pp. 282-309). Oxford: Blackwell.

DeLoache, Judy (2004). Becoming symbol-minded. Trends in Cognitive Sciences, 8, 66-70.

Desrochers, Stephan, Paul Morissette, \& Marcelle Ricard (1995). Two perspectives on pointing in infancy. In Chris Moore \& Philip J. Dunham (Eds.), Joint attention: Its origins and role in development (pp. 85-101). Hillsdale, NJ: Lawrence Erlbaum.

Franco, Fabia \& George Butterworth (1996). Pointing and social awareness: Declaring and requesting in the second year. Journal of Child Language, 23 (2), 307-336.

Goldin-Meadow, Susan (2003). The resilience of language: What gesture creation in deaf children can tell us about how all children learn language. New York: Psychology Press.

Gomez, Juan C., Encarnacion Sarria, \& Javier Tamarit (1993). The comparative study of early communication and theories of mind: Ontogeny, phylogeny, and pathology. In Simon Baron-Cohen, Helen Tager-Flusberg, et al. (Eds.), Understanding other minds: Perspectives from autism (pp 397-426). New York: Oxford University Press.

Goodwyn, Susan \& Laura Acredolo (1993). Symbolic gesture versus word: Is there a modality advantage for onset of symbol use? Child Development, 64, 688-701.

Grice, Paul (1957). Meaning. The Philosophical Review, 64, 377-388.

Harris, Paul \& Robert Kavanaugh (1993). Young children's understanding of pretense. Monographs of the Society for Research in Child Development, 58 (1) [231], v-92.

Iverson, Jana, Olga Capirci, \& Maria Caselli (1994). From communication to language in two modalities. Cognitive Development, 9, 23-43.

Iverson, Jana \& Susan Goldin-Meadow (2005) Gesture paves the way for language development. Psychological Science, 16 (5), 367-371.

Liszkowski, Ulf, Malinda Carpenter, Anne Henning, Tricia Striano, \& Michael Tomasello (2004). Twelve-month-olds point to share attention and interest. Developmental Science, 7 (3), 297-307.

Liszkowski, Ulf, Malinda Carpenter, Tricia Striano, \& Michael Tomasello (2006). Twelve- and 18-month-olds point to provide information for others. Journal of Cognition and Development, 7 (2), 173-187.

Liszkowski, Ulf, Malinda Carpenter, \& Michael Tomasello (2007a). Reference and attitude in infant pointing. Journal of Child Language, 34, 1-20.

Liszkowski, Ulf, Malinda Carpenter, \& Michael Tomasello (2007b). Pointing out new news, old news, and absent referents at 12 months of age. Developmental Science, 10 (2). F1-F7.

Liszkowski, Ulf, Konstanze Albrecht, Malinda Carpenter, \& Michael Tomasello (2008). Infants' visual and auditory communication when a partner is or is not visually attending. Infant Behavior and Development, 31, 157-167.

Liszkowski, Ulf, Malinda Carpenter, \& Michael Tomasello (submitted). Twelve-month-olds communicate helpfully, and appropriately for knowledgeable and ignorant partners. 
Liszkowski, Ulf, Marie Schäfer, Malinda Carpenter, \& Michael Tomasello (submitted). Prelinguistic infants refer to absent entities.

Lock, Andrew, Andrew Young, Valerie Service, \& Paul Chandler (1990). Some observations on the origins of the pointing gesture. In Virginia Volterra \& Carol J. Erting (Eds.), From gesture to language in hearing and deaf children (pp. 42-55). Berlin: Springer.

Moore, Chris \& Babara D'Entremont (2001). Developmental changes in pointing as a function of attentional focus. Journal of Cognition \& Development, 2, 109-129.

Namy, Laura, Aimee Campbell, \& Michael Tomasello (2004). The changing role of iconicity in non-verbal symbol learning: A U-shaped trajectory in the acquisition of arbitrary gestures. Journal of Cognition and Development, 5 (1), 37-57.

Pizzuto, Elena \& Micaela Capobianco (2005). The link and differences between deixis and symbols in children's early gestural-vocal system. Gesture, 5 (1/2), 179-199.

Rakoczy, Hannes, Michael Tomasello, \& Tricia Striano (2004). Young children know that trying is not pretending - a test of the "behaving-as-if" construal of children's early concept of "pretense". Developmental Psychology, 40 (3), 388-399.

Senghas, Ann, Sotaro Kita, \& Asli Özyürek (2004). Children creating core properties of language: Evidence from an emerging sign language in Nicaragua. Science, 305 (5691), 1779-1782.

Sperber, Dan \& Deirdre Wilson (1986/1995). Relevance: Communication and cognition. Oxford: Blackwell.

Tomasello, Michael, Malinda Carpenter, \& Ulf Liszkowski (2007). A new look at infant pointing. Child Development, 78, 705-722.

Tomasello, Michael (2006). Why don't apes point? In Nick Enfield \& Steve Levinson (Eds.), The roots of human sociality: Culture, cognition, and interaction. Oxford: Berg.

Werner, Heinz \& Bernhard Kaplan (1963). Symbol formation: An organismic-developmental approach to language and the expression of thought. New York: Wiley.

\section{Author's address}

Ulf Liszkowski

PO Box 310

6500 AH Nijmegen

The Netherlands

ulf.liszkowski@mpi.nl

\section{About the author}

Ulf Liszkowski received his PhD in Psychology from the University of Leipzig, Germany. He is head of the Max-Planck Independent Junior Research Group Communication Before Language at the MPI for Psycholinguistics in Nijmegen, The Netherlands. His research addresses infants' prelinguistic communication and their social and cognitive development. 